\title{
Effect of the interaction between atorvastatin and selective serotonin reuptake inhibitors on the blood redox equilibrium
}

\author{
MARIOLA HERBET, MONIKA GAWROŃSKA-GRZYWACZ, \\ MAGDALENA IZDEBSKA and IWONA PIĄTKOWSKA-CHMIEL
}

\author{
Chair and Department of Toxicology, Medical University of Lublin, 20-093 Lublin, Poland
}

Received November 4, 2015; Accepted January 27, 2016

DOI: $10.3892 /$ etm.2016.3794

\begin{abstract}
Cardiovascular disease and depression often occur simultaneously in the same patient. Long-term polypharmacotherapy can lead to drug-induced oxidative stress. Data concerning the effects of concomitant treatment with atorvastatin and selective serotonin reuptake inhibitors (SSRIs) are lacking. The aim of the present study was to examine oxidative stress parameters in the blood of rats after 28 days treatment with atorvastatin combined with fluoxetine or paroxetine. The study was carried out on male Wistar rats weighing 200-250 g. Aqueous solutions of atorvastatin $(10 \mathrm{mg} / \mathrm{kg})$, fluoxetine $(10 \mathrm{mg} / \mathrm{kg})$ and paroxetine $(10 \mathrm{mg} / \mathrm{kg})$ were injected once a day for 28 days, separately or concomitantly. The activity of glutathione peroxidase (GPX) was determined in the whole blood, whereas the activity of glutathione reductase (GR) and the total antioxidant status (TAS) were determined in the serum. The results demonstrated that concomitant administration of atorvastatin with fluoxetine caused an increase in the GPX activity and the TAS. Atorvastatin administered to rats with paroxetine increased the activities of GPX and GR. In the groups of rats receiving atorvastatin or SSRIs separately, no statistically significant changes in the investigated parameters were observed. The changes that were detected may indicate an increase in endogenous antioxidant levels during the concomitant application of atorvastatin with SSRIs and thus a drug-drug interaction having an effect on the blood redox equilibrium.
\end{abstract}

\section{Introduction}

Cardiovascular diseases and depression are very prevalent among the general population and often occur simultaneously in the same patient (1). The association between depression

Correspondence to: Dr Mariola Herbet, Chair and Department of Toxicology, Medical University of Lublin, 8 Chodzki Street, 20-093 Lublin, Poland

E-mail: mariola.herbet@umlub.pl

Key words: oxidative stress parameters, atorvastatin, fluoxetine, paroxetine, rat and heart disease is two directional. Depression is regarded as a risk factor for coronary heart disease and acute cardiovascular sequelae, including myocardial infarction and heart failure (2). Conversely, patients with heart disease exhibit increased episodes of depression $(3,4)$. Moreover, morbidity and mortality rates in patients with depression and cardiovascular disease have been found to be significantly higher than those in patients with cardiovascular disease without depression (4). It has been observed that patients who experienced a myocardial infarction and became depressed had a 5-fold increase in mortality rate during the 6 months after the infarction (5). Therefore, the effective treatment of depression in patients with cardiovascular disease is vital and requires the simultaneous use of multiple medications.

Clinical trials have indicated that there is an association between cardiovascular improvement and the degree by which low-density lipoprotein cholesterol (LDL-C) levels are reduced (6). The reduction of high blood cholesterol levels is a crucial purpose of pharmacotherapy. The most effective lipid-lowering drugs are statins, also known as 3-hydroxy-3-methylglutaryl-CoA reductase inhibitors. A drug of the statin class, atorvastatin, reduces the risk of cardiovascular events by reducing the total cholesterol, LDL-C and B apolipoprotein levels in serum (7). Atorvastatin is also effective in treating and preventing atherosclerosis, and affects all stages of atherosclerotic plaque formation (8).

Selective serotonin reuptake inhibitors (SSRIs) are the most frequently prescribed class of antidepressants. SSRIs are generally recommended for use in patients with cardiac disease and may also have a protective effect against myocardial infarction. Several studies have documented the cardiovascular effects of fluoxetine treatment (9-11). The SSRI fluoxetine appears to be safe for use in the treatment of depressed patients with cardiac disease and does not cause orthostatic hypotension or slow conduction (10). Another SSRI, paroxetine, is one of the most well-known and effective SSRIs $(12,13)$. According to Nelson et al, depressed patients with ischemic heart disease can be treated with paroxetine effectively and safely as paroxetine has no consistent significant effects on the pulse or blood pressure, and the authors suggest that paroxetine is as effective as nortriptyline but less likely to produce serious side effects (5).

Atorvastatin undergoes extensive microsomal metabolism by the cytochrome P450, CYP3A4 isoenzyme (14). 
Co-administration of drugs that interact with the CYP450 system may significantly affect the plasma concentrations and potential toxicity of atorvastatin. Fluoxetine and its circulating metabolite norfluoxetine constitute a complex multiple inhibitor system, causing reversible or time-dependent inhibition of CYP3A4, and also CYP2D6 and CYP2C19 in vitro (15). Paroxetine is extensively metabolized and is a mechanism-based inhibitor of CYP2D6 and CYP3A. This inhibition most likely involves the irreversible binding of a paroxetine-reactive metabolite to the heme complex in the P450 enzyme (16). Long-term polypharmacotherapy can lead to increased side effects of drugs and may cause an oxidation-reduction imbalance followed by the generation of reactive oxygen species (ROS) (17-19). ROS are produced intracellularly through multiple mechanisms, and the sources of ROS in cells include CYP450 CYP and NADPH oxidase, with NADPH oxidase complexes being the major producers of ROS in cells. Biochemical and pharmacokinetic studies have revealed that during the biotransformation of drugs, reactive metabolites capable of directly reducing molecular oxygen to generate ROS are often produced. Cytochrome P450 is a superfamily of heme-thiolate proteins that are, in general, the terminal oxidase enzymes in an electron transfer chain that delivers an electron to an oxygen molecule $(17,18,20)$. During phase I metabolism, atorvastatin is extensively metabolized by the monooxygenase CYP3A4, with the insertion of an oxygen atom into the atorvastatin molecule to form $o$ - and $p$-hydroxyatorvastatin, while the other oxygen atom of molecular oxygen is reduced to water with the concomitant oxidation of NADPH; however, premature and incomplete reduction may occur, to generate the superoxide radical $\left(\mathrm{O}_{2}{ }^{-}\right)(20,21)$. SSRIs cause reversible or time-dependent inhibition of CYP3A4 and also CYP2D6 which could affect the metabolism of atorvastatin (22).

Drug metabolism generates ROS and reactive metabolites as by-products; however, cells have a defense mechanism for the removal of free radicals to attenuate intracellular damage and ameliorate the harmful effects of ROS $(18,23)$. Drug interactions may alter cell metabolism causing the cellular antioxidant capacity to be exceeded, possibly resulting in increased oxidative stress. Drug-induced oxidative stress has been implicated as a mechanism of toxicity in numerous tissues (18). Therefore, any potential drug interactions and adverse effects require close monitoring.

To the best of our knowledge, there are no published data concerning the effects of concomitant treatment with atorvastatin and SSRIs on redox imbalance. The present study aimed to assess antioxidant defense parameters in the blood of rats after 28 days treatment with atorvastatin in combination with fluoxetine or paroxetine. The activity of glutathione peroxidase (GPX) was determined in whole blood and the glutathione reductase (GR) activity and total antioxidant status (TAS) were determined in serum.

\section{Materials and methods}

Drugs and chemicals. The following substances were used in the study: Atorvastatin (atorvastatin calcium salt trihydrate, Atorvastatin Ranbaxy; Ranbaxy, Warsaw, Poland), fluoxetine (fluoxetine hydrochloride, Fluoksetyna Egis;
Egis Pharmaceuticals PLC, Keresztúri, Hungary), paroxetine (paroxetine hydrochloride semihydrate, Seroxat; GlaxoSmithKline, Brentford, UK) and aqua pro injectione (Baxter, Warsaw, Poland). Ready-made diagnostic kits (Randox Laboratories Ltd., Crumlin, UK) were used to determine the GPX (RS505) and GR (GR2368) activities and the TAS (NX2332).

Animals. The present study was carried out on 48 male Wistar rats (age, 6-weeks-old; initial weight, 200-250 g) that were obtained from a licensed breeder (Breeding of Laboratory Animals, Zbigniew Lipiec, Brwinów, Poland). The animals were kept at room temperature $\left(20 \pm 2^{\circ} \mathrm{C}\right)$ under a $12 \mathrm{~h}$ day $/ 12 \mathrm{~h}$ night cycle under constant environmental conditions $(55 \pm 10 \%$ humidity and noise). They had access to food and water ad libitum. The study was approved by the Ethics Committee on Animal Experimentation of the Medical University of Lublin (Lublin, Poland).

Experimental procedures. Aqueous solutions of atorvastatin $(10 \mathrm{mg} / \mathrm{kg})$, fluoxetine $(10 \mathrm{mg} / \mathrm{kg})$ and paroxetine $(10 \mathrm{mg} / \mathrm{kg})$ were prepared ex tempore and injected intraperitoneally once a day for 28 days, individually or combined, in a constant volume of $0.5 \mathrm{ml} / 100 \mathrm{~g}$ of body weight. The doses were selected based on those reported in the literature and in our previous experiments, which demonstrated that the aforementioned doses of statin and antidepressant drugs are effective (24-26). The rats in the control group received the same amounts of aqua pro injectione at the same time points. Six groups of rats, each consisting of 8 animals, were treated in the following order: i) Control group, treated with aqua pro injection; ii) atorvastatin group; iii) fluoxetine group; iv) paroxetine group; v) atorvastatin plus fluoxetine group (treated with atorvastatin followed by fluoxetine 5 min later); and vi) atorvastatin plus paroxetine group (treated with atorvastatin followed by paroxetine 5 min later). The studied drugs were administered to rats in the aforementioned effective doses. At $24 \mathrm{~h}$ after the last injection, the animals were decapitated and blood samples were taken and divided as follows: One portion was stored in a heparin tube (whole blood) and the other was left to clot. Animals decapitation was performed in accordance with European standards related to the experimental studies on animal models. According to the guidelines, handling of animals and injection of sedatives or anaesthetics prior to decapitation may increase stress prior to euthanasia and is therefore not considered good for the welfare of the animal. In the present study, the animals were not anesthetized prior to decapitation.

The whole heparinized blood (for quantitative in vitro determination of GPX in whole blood) was tested to estimate the GPX activity by kinetic methods, using the aforementioned kit. The method of determination was based on a method previously described by Paglia and Valentine (23). In this method, GPX catalyzes the oxidation of glutathione by cumene hydroperoxide, and in the presence of GR and NADPH the oxidized glutathione (GSSG) is immediately converted to the reduced form with a concomitant oxidation of NADPH to NADP ${ }^{+}$. The reduction in absorbance at $340 \mathrm{~nm}$ was measured.

In the portion of the blood that was allowed to clot, the serum fraction was removed and the GR activity and TAS in 
the serum fraction were determined by kinetic methods using the aforementioned kits. The GR activity assay is based on the reduction of GSSG catalyzed by GR in the presence of NADPH, which is oxidized to $\mathrm{NADP}^{+}$. The reduction in absorbance at $340 \mathrm{~nm}$ was measured. The TAS determination method consists of incubating 2,2'-azino-di-(3-ethylbenzthiazoline sulfonate (ABTS) with a peroxidase (metmyoglobin) and $\mathrm{H}_{2} \mathrm{O}_{2}$ to produce $\mathrm{ABTS}^{+}$. This radical cation has a relatively stable blue-green color, which is measured at $600 \mathrm{~nm}$.

All procedures were performed according to the instructions supplied with the respective kits.

Statistical analysis. Results are expressed as the mean \pm standard error of the mean. Groups in which single drugs were administered were compared with the control group and one-way analysis of variance (ANOVA) was used (followed by Dunnett's test). Double-drug groups were compared with the control and single-drug groups and two-way ANOVA with Tukey's post hoc test was used to determine statistical significance. $\mathrm{P}<0.05$ was considered to indicate a statistically significant difference.

\section{Results}

GPX activity. The conducted experiments demonstrated that the 28-day concomitant administration of atorvastatin $(10 \mathrm{mg} / \mathrm{kg})$ with fluoxetine $(10 \mathrm{mg} / \mathrm{kg})$ caused an increase in the GPX activity in the blood of the rats compared with that in the control group and with the group of animals receiving only fluoxetine $(\mathrm{P}<0.05$; Fig. 1). The combined administration of atorvastatin with paroxetine caused a significant increase in the activity of GPX compared with the control group. In animal groups receiving atorvastatin or an SSRI separately, the activity of GPX remained unaffected compared with the control.

GR activity. Atorvastatin $(10 \mathrm{mg} / \mathrm{kg})$ administered to rats with paroxetine $(10 \mathrm{mg} / \mathrm{kg})$ increased the activity of GR compared with that of the control group ( $\mathrm{P}<0.05$; Fig. 2$)$. In the groups of rats receiving atorvastatin or an SSRI separately and in the group treated with atorvastatin and fluoxetine simultaneously the GR relevant activity did not change from that in the control group.

TAS of blood. The results of the TAS assay indicate that the 4-week combined treatment with atorvastatin and fluoxetine caused an increase in the TAS compared with that of the control group and the group receiving atorvastatin alone ( $\mathrm{P}<0.05$; Fig. 3), although the standard error of the mean was high. In the remaining groups, no statistically significant changes in TAS level were observed.

\section{Discussion}

The formation of chemically reactive metabolites of drugs is associated with numerous adverse drug reactions $(18,19)$. Reactive metabolites may react with cellular proteins, lipids and nucleic acids and thus cause oxidative stress. Atorvastatin is extensively metabolized in the gut and liver by oxidation, lactonization and glucuronidation. Metabolism

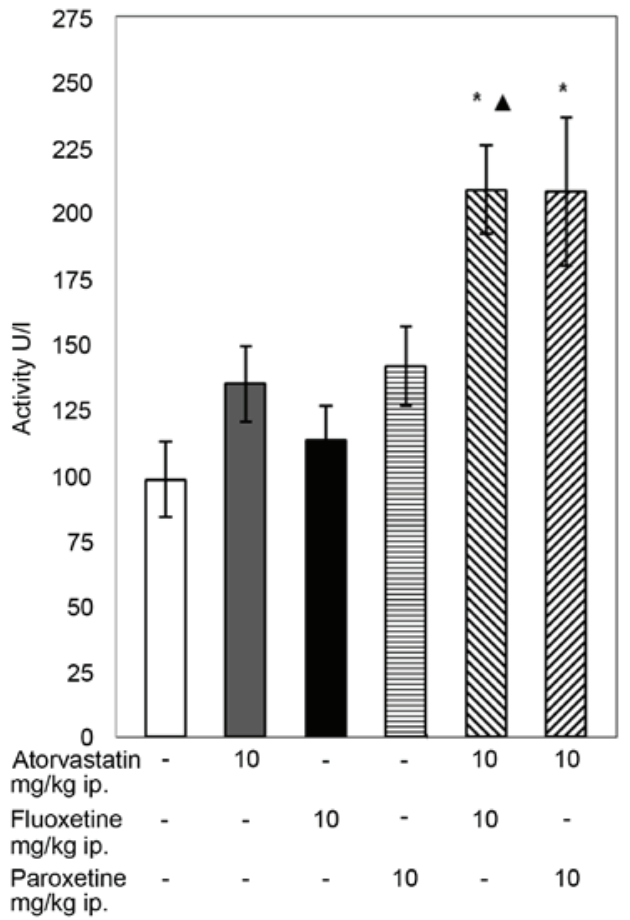

Figure 1. Impact of 28-day intraperitoneal (i.p.) administration of atorvastatin $(10 \mathrm{mg} / \mathrm{kg})$ and fluoxetine $(10 \mathrm{mg} / \mathrm{kg})$ or paroxetine $(10 \mathrm{mg} / \mathrm{kg})$ on the activity of glutathione peroxidase in the blood of rats. The results are presented as mean \pm standard error of the mean. Data were analyzed by one-way analysis of variance (ANOVA) followed by Dunnett's test (single-drug groups vs. control) and two-way ANOVA with Tukey's post hoc test (double-drug groups vs. the respective single-drug groups). ${ }^{*} \mathrm{P}<0.05$ vs. the control group; ${ }^{\wedge} \mathrm{P}<0.05$ vs. the fluoxetine group.

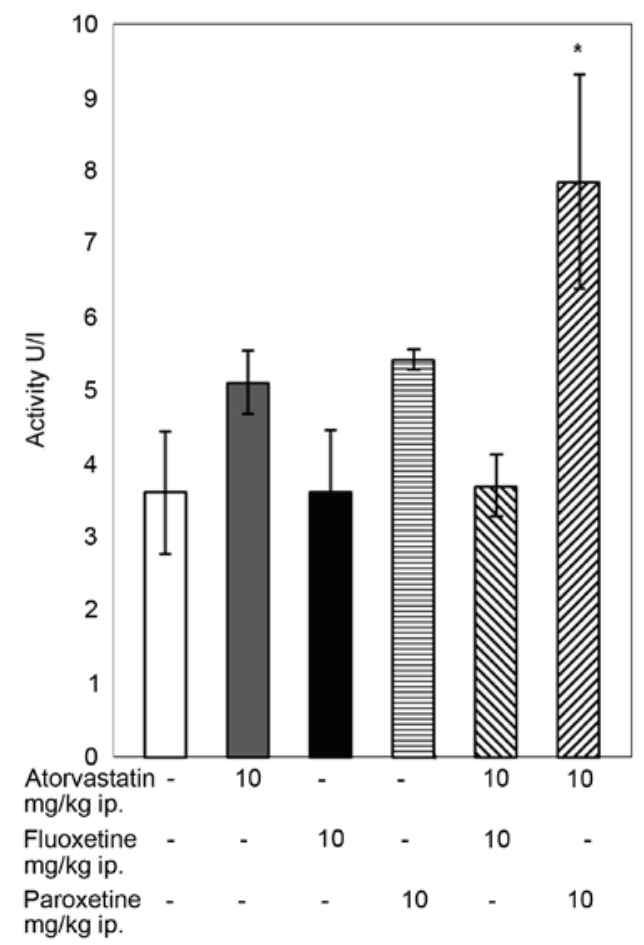

Figure 2. Impact of 28-day intraperitoneal administration of atorvastatin $(10 \mathrm{mg} / \mathrm{kg})$ and fluoxetine $(10 \mathrm{mg} / \mathrm{kg})$ or paroxetine $(10 \mathrm{mg} / \mathrm{kg})$ on the activity of glutathione reductase in the serum of rats. The results are presented as mean \pm standard error of mean. Data were analyzed by one-way analysis of variance (ANOVA) followed by Dunnett's test (single-drug groups vs. control) and two-way ANOVA with Tukey's post hoc test (double-drug groups vs. the respective single-drug groups). ${ }^{*} \mathrm{P}<0.05$ vs. the control group. 


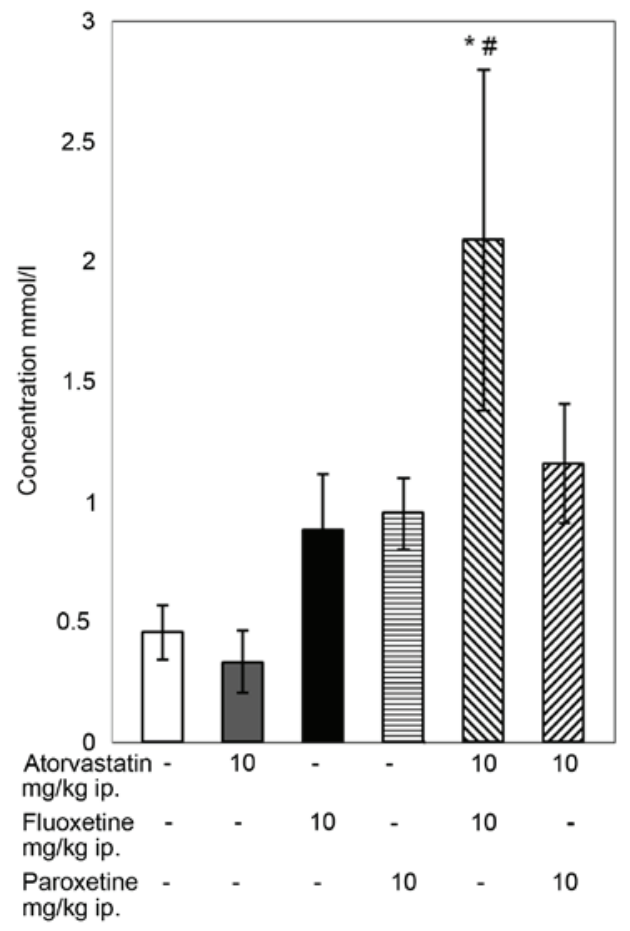

Figure 3. Impact of 28-day intraperitoneal administration of atorvastatin $(10 \mathrm{mg} / \mathrm{kg})$ and fluoxetine $(10 \mathrm{mg} / \mathrm{kg})$ or paroxetine $(10 \mathrm{mg} / \mathrm{kg})$ on the total antioxidant status in the serum of rats. The results are presented as mean \pm standard error of mean. Data were analyzed by one-way analysis of variance (ANOVA) followed by Dunnett's test (single-drug groups vs. control) and two-way ANOVA with Tukey's post hoc test (double-drug groups vs. the respective single-drug groups). ${ }^{*} \mathrm{P}<0.05$ vs. the control group; ${ }^{\#} \mathrm{P}<0.05$ vs. the atorvastatin group.

in the liver produces two active hydroxy metabolites, $o$-hydroxyatorvastatin and $p$-hydroxyatorvastatin (21). During the bioactivation of atorvastatin, the reduction of molecular oxygen by reactive metabolites may generate $\operatorname{ROS}(17,8,20)$. The risk of ROS being generated increases during simultaneous treatment with several drugs metabolized by cytochrome P450 and this can lead to redox imbalance (17). Oxidative stress causes cell damage through a number of mechanisms including lipid peroxidation, protein oxidation, DNA oxidation and mitochondrial damage. This impairment of cellular function can result in cell death and possible organ failure $(27,28)$. However, cells contain antioxidant enzymes that function to maintain the redox status.

In the present study, the combined administration of atorvastatin with fluoxetine caused an increase of GPX activity and did not influence GR activity. GPX is one of the body's most potent antioxidant defense agents. Its purpose is to balance pro-oxidative and antioxidant actions and to prevent the excessive accumulation of ROS in the body. A change in the activity of this enzyme may reflect the impairment of antioxidant activity in the blood and can be useful in monitoring the effects of treatment (23). In the present study, the increase of GPX activity indicated that endogenous antioxidant levels were increased. This might indicate that the production of free radicals was increased following the concomitant application of atorvastatin with fluoxetine. Changes in GPX activity have been observed in animals exposed to pro-oxidative drug action (18). In our previous study, increases in the activity of both GPX and GR were observed after the combined 14-day administration of rosuvastatin with fluoxetine to rats (24). In the present study, the simultaneous application of atorvastatin with fluoxetine caused an increase in the TAS, although with a high error of the mean. In the body, the total antioxidant system consists of multiple components providing protection against the molecular damage of cell structures. The TAS is defined as the ability of the serum to quench free radical production, and is determined in order to indicate the efficiency of cellular antioxidant mechanisms. A reduction in the TAS suggests an increase in the number of oxygen free radicals and a reduction of the activity of the antioxidant defense system. The TAS also indicates the antioxidant potential of drugs and can indicate whether a new treatment is detrimental to the body's anti-oxidation system. In the present study, an increase of the TAS following the co-administration of atorvastatin with fluoxetine is probably associated with metabolic mobilization and may indicate that the amounts of endogenous antioxidants were increased. Our previous study carried out on rats treated simultaneously with rosuvastatin and fluoxetine for 14 days revealed a reduction in the TAS (24). This difference may result from the shared metabolism of rosuvastatin and fluoxetine; these drugs are biotransformed by cytochrome P450 isoenzyme CYP2C9, while atorvastatin is metabolized primarily by isoenzyme CYP3A4 $(15,21,28)$. It is also important to note that different durations of treatment were applied (14 days in the previous study, and 28 days in the current study). The present study was a preliminary study, and did not include the monitoring of transcription factors, such as Nrf2, that are responsible for activating the antioxidant capacity of cells.

Combined administration of atorvastatin with paroxetine caused an increase in the activity of both GPX and GR (only in comparison with the control group) and did not affect the TAS. GR is closely associated with GPX and plays an important role in protecting cells against oxidative damage by increasing the level of reduced glutathione in the process of aerobic glycolysis (29). The increased activity of GR may be influenced by the reduced form of glutathione, and potentially indicates that there is an increased activity of the enzyme system that prevents oxidation. Similar results were obtained in our previous study, in which rats were treated with rosuvastatin and paroxetine simultaneously for 14 days (25).

The present study revealed no significant changes in the activity of GPX and GR enzymes or in the TAS in rats treated with only atorvastatin. However, a number of studies support the role of atorvastatin in the maintenance of the antioxidant status (30-32). The possible antioxidant mechanisms of atorvastatin include reducing the generation of ROS by the inhibition of vascular $\mathrm{NAD}(\mathrm{P}) \mathrm{H}$ oxidase, modifying redox homeostasis in LDL particles, modulating RNA expression, increasing the synthesis of nitric oxide in the vasculature and binding to the surface phospholipids of lipoproteins $(30,33)$. In the current study, no statistically significant changes concerning the oxidative stress parameters in the groups of rats receiving antidepressants individually were noted. Previous studies suggest that SSRIs have a beneficial effect in maintaining the oxidative-reductive balance $(34,35)$. The potentially favorable antioxidant effect of fluoxetine may be mediated by three mechanisms: Inhibition of lipid peroxidation, increase of glutaminergic transmission and reduction of immune and inflammatory components that 
favor the generation of ROS (36). Similar results, which did not reveal any crucial changes in oxidative stress parameters in rats receiving fluoxetine for 14 days, were obtained in a previous study (24). Other research indicates that the antidepressive effect of paroxetine is at least partially associated with the attenuation of oxidative stress imbalance (37).

An increase in GPX activity in the rats receiving atorvastatin with fluoxetine may be associated with the defense mechanism against the increased ROS load. An increase in the TAS is probably connected with the metabolic mobilization of the rats treated with atorvastatin plus fluoxetine, and may indicate an increasing amount of endogenous antioxidants. Concurrent increases in the activity of GPX and GR are likely to be due to the augmentation of free oxygen radical production during the simultaneous administration of atorvastatin with paroxetine. The present study was a preliminary study, aiming to assess antioxidant defense parameters in the blood of rats by assaying the activity of antioxidant enzymes and TAS level. In the future, this research may be expanded, for example, to determine the level of glutathione.

\section{References}

1. Frasure-Smith N, Lespérance F, Irwin MR, Talajic M and Pollock BG: The relationships among heart rate variability, inflammatory markers and depression in coronary heart disease patients. Brain Behav Immun 23: 1140-1147, 2009.

2. Thombs BD, Bass EB, Ford DE, Stewart KJ, Tsilidis KK, Patel U, Fauerbach JA, Bush DE and Ziegelstein RC: Prevalence of depression in survivors of acute myocardial infarction. J Gen Intern Med 21: 30-38, 2006.

3. Mathews MJ, Mathews EH and Liebenberg L: The mechanisms by which antidepressants may reduce coronary heart disease risk. BMC Cardiovasc Disord 15: 82, 2015.

4. Nemeroff CB and Goldschmidt-Clermont PJ: Heartache and heartbreak-the link between depression and cardiovascular disease. Nat Rev Cardiol 9: 526-539, 2012.

5. Nelson JC, Kennedy JS, Pollock BG, Laghrissi-Thode F, Narayan M, Nobler MS, Robin DW, Gergel I, McCafferty J and Roose S: Treatment of major depression with nortriptyline and paroxetine in patients with ischemic heart disease. Am J Psychiatry 156: 1024-1028, 1999.

6. Sirtori CR and Fumagalli R: LDL-cholesterol lowering or HDL-cholesterol raising for cardiovascular prevention. A lesson from cholesterol turnover studies and others. Atherosclerosis 186: 1-11, 2006.

7. Kobayashi J, Maruyama T, Masuda M and Shinomiya M: Effect of atorvastatin treatment on lipoprotein lipase mass in the pre-heparin plasma in Japanese hyperlipidemic subjects. Clin Chim Acta 314 261-264, 2001.

8. Eshtehardi P, McDaniel MC, Dhawan SS, Binongo JN, Krishnan SK, Golub L, Corban MT, Raggi P, Quyyumi AA and Samady H: Effect of intensive atorvastatin therapy on coronary atherosclerosis progression, composition, arterial remodeling, and microvascular function. J Invasive Cardiol 24: 522-529, 2012.

9. Baumeister H, Hutter $\mathrm{N}$ and Bengel J: Psychological and pharmacological interventions for depression in patients with coronary artery disease. Cochrane Database Syst Rev: CD008012, 2011.

10. Roose SP: Treatment of depression in patients with heart disease. Biol Psychiatry 54: 262-268, 2003.

11. Roose SP and Miyazaki M: Pharmacological treatment of depression in patients with heart disease. Psychosom Med 67 (Suppl 1): S54-S57, 2005.

12. Sanchez C, Reines EH and Montgomery SA: A comparative review of escitalopram, paroxetine, and sertaline: Are they all alike? Int Clin Psychopharmacol 29: 185-196, 2014.

13. Vaswani M, Linda FK and Ramesh S: Role of selective serotonin reuptake inhibitors in psychiatric disorders: A comprehensive review. Prog Neuropsychopharmacol Biol Psychiatry 27: 85-102, 2003.

14. Bolego C, Baetta R, Bellosta S, Corsini A and Paoletti R: Safety considerations for statins. Curr Opin Lipidol 13: 637-644, 2002.
15. Andrade C: Selective serotonin reuptake inhibitor drug interactions in patients receiving statins. J Clin Psychiatry 75: e95-e99, 2014.

16. Jornil J, Jensen KG, Larsen F and Linnet K: Identification of cytochrome P450 isoforms involved in the metabolism of paroxetine and estimation of their importance for human paroxetine metabolism using a population-based simulator. Drug Metab Dispos 38: 376-385, 2010

17. Bhattacharyya S, Sinha K and Sil PC: Cytochrome P450s: Mechanisms and biological implications in drug metabolism and its interaction with oxidative stress. Curr Drug Metab 15: 719-742, 2014.

18. Deavall DG, Martin EA, Horner JM and Roberts R: Drug-induced oxidative stress and toxicity. J Toxicol 2012: 645460, 2012.

19. Cunha-Oliveira T, Rego AC and Oliveira CR: Oxidative stress and drugs of abuse: An update. Mini-Rev Org Chem 10: 000-000, 2013.

20. Guengerich FP: Cytochrome P450s and other enzymes in drug metabolism and toxicity. AAPS J 8: E101-E111, 2006.

21. Lins RL, Matthys KE, Verpooten GA, Peeters PC, Dratwa M, Stolear JC and Lameire NH: Pharmacokinetics of atorvastatin and its metabolites after single and multiple dosing in hypercholesterolaemic haemodialysis patients. Nephrol Dial Transplant 18: 967-976, 2003.

22. Li X, Fang P, Mai J, Choi ET, Wang H and Yang XF: Targeting mitochondrial reactive oxygen species as novel therapy for inflammatory diseases and cancers. J Hematol Oncol 6: 19, 2013.

23. Paglia DE and Valentine WN: Studies on the quantitative and qualitative characterization of erythrocyte glutathione peroxidase. J Lab Clin Med 70: 158-169, 1967.

24. Herbet M, Gawrońska-Grzywacz M and Jagiełło-Wójtowicz E: Evaluation of selected biochemical parameters of oxidative stress in rats pretreated with rosuvastatin and fluoxetine. Acta Pol Pharm 72: 261-265, 2015.

25. Herbet M, Izdebska M, Piątkowska-Chmiel I, Poleszak E and Jagiełło-Wójtowicz E: Estimation of oxidative stress parameters in rats after simultaneous administration of rosuvastatin with antidepressants. Pharmacol Rep 68: 172-176: 2016.

26. Almeida J, Duarte JO, Oliveira LA, Crestani CC: Effects of nitric oxide synthesis inhibitor or fluoxetine treatment on depressionlike state and cardiovascular changes induced by chronic variable stress in rats. Stress 18: 462-474.

27. Park BK, Laverty H, Srivastava A, Antoine DJ, Naisbitt D and Williams DP: Drug bioactivation and protein adduct formation in the pathogenesis of drug-induced toxicity. Chem Biol Interact 192: 30-36, 2011.

28. Webb HM, Regan S, Antoine DJ, Lane N, Walsh RJ, Srivastava A, Starkey-Lewis P, Benson C, Williams DP, Laverty H, et al: Drug Bioactivation and Oxidative Stress. In: Encyclopedia of Drug Metabolism and Interactions. Lyubimov AV (ed.) John Wiley \& Sons, New York: 1-31, 2012

29. Chang JC, van der Hoeven LH and Haddox CH: Glutathione reductase in the red blood cells. Ann Clin Lab Sci 8: 23-29, 1978.

30. Koksal M, Eren MA, Turan MN and Sabuncu T: The effects of atorvastatin and rosuvastatin on oxidative stress in diabetic patients. Eur J Intern Med 22: 249-253, 2011.

31. Bloom HL, Shukrullah I, Veledar E, Gutmann R, London B and Dudley SC: Statins decrease oxidative stress and ICD therapies. Cardiol Res Pract 2010: 253803, 2010.

32. Nagila A, Permpongpaiboon T, Tantrarongroj S, Porapakkham P, Chinwattana K, Deakin S and Porntadavity S: Effect of atorvastatin on paraoxonase1 (PON1) and oxidative status. Pharmacol Rep 61: 892-898, 2009.

33. Cangemi R, Loffredo L, Carnevale R, Perri L, Patrizi MP, Sanguigni V, Pignatelli P and Violi F: Early decrease of oxidative stress by atorvastatin in hypercholesterolaemic patients: Effect on circulating vitamin E. Eur Heart J 29: 54-62, 2008.

34. Abdel-Salam OME, Youssef Morsy SM and Sleem AA: The effect of different antidepressant drugs on oxidative stress after lipopolysaccharide administration in mice. EXCLI J 10: 290-302, 2011.

35. Zafir A and Banu N: Antioxidant potential of fluoxetine in comparison to Curcuma longa in restraint-stressed rats. Eur J Pharmacol 572: 23-31, 2007.

36. Novío S, Núñez MJ, Amigo G and Freire-Garabal M: Effects of fluoxetine on the oxidative status of peripheral blood leucocytes of restraint-stressed mice. Basic Clin Pharmacol Toxicol 109: 365-371, 2011.

37. Qiu HM, Yang JX, Wu XH, Li N, Liu D, Wang LJ, Qin LJ and Zhou QX: Antidepressive effect of paroxetine in a rat model: Upregulating expression of serotonin and norepinephrine transporter. Neuroreport 24: 520-525, 2013. 